\title{
Ayushman Bharat and Universal Health Coverage in India: Is our approach ethical?
}

\author{
SOHAM D BHADURI
}

\begin{abstract}
The Ayushman Bharat Mission with its two inter-linked components, namely Health and Wellness centres and the Pradhan Mantri Jan Arogya Yojana, has been proposed as India's prime instrument for achieving universal health coverage. The insurance component of the mission has received priority over the primary healthcare component. Apart from efficiency reasons, there are strong ethical grounds to prioritize the primary care component over its insurance counterpart. There is also a need to consider the ethical dimensions of publicly financed health insurance in India. A robust priority setting process should guide the roll-out of universal health coverage. This is particularly crucial since a large scale expansion of PMJAY to the non-poor population is being currently envisaged by the government.
\end{abstract}

Keywords: Equality, universal health coverage, public health ethics, health financing, primary health care, Ayushman Bharat

\section{Introduction}

For over a decade, universal health coverage (UHC) has featured prominently on the global health agenda. There has been an increasing emphasis on UHC for low- and lowmiddle income countries (LMIC) in view of its ability to contribute to overall economic and social well-being. The archetypal LMIC context is characterised by limited fiscal space and resources for health and other social sectors, weak political accountability, and limited stakeholder participation in many policy decisions. A natural consequence in such contexts is that economic, political, and implementational

Author: Soham D. Bhaduri (soham.bhaduri@gmail.com), Physician and Public Health Commentator, and Editor, The Indian Practitioner. 'Parijat', Barku Pada Road, Ambernath - East, Thane District, 421 501, INDIA.

To cite: Bhaduri SD. Ayushman Bharat and Universal Health Coverage in India: Is our approach ethical? Indian J Med Ethics. 2021 Jul-Sep; 6(3) NS: 238-241. DOI: 10.20529/IJME.2021.016.

Published online first on March 8, 2021.

Manuscript editor:Vijayaprasad Gopichandran

Peer Reviewer: Nobhojit Roy

(c) Indian Journal of Medical Ethics 2021 expediency become the prime drivers of health policy decisions. Questions of ethics and fairness are often shifted to the back-seat, unless of course they present glaring concerns of tremendous magnitude.

In India, the Ayushman Bharat Mission has been advanced as the prime instrument for achieving UHC. It has two interlinked components, namely Health and Wellness (H \&W) centres and the Pradhan Mantri Jan Arogya Yojana (PMJAY). The former aims to upgrade sub-centres and primary health centres to deliver comprehensive primary healthcare to the population. The latter is a national, publicly-funded health insurance (PFHI) scheme covering secondary and tertiary care hospitalisation for the most deprived 40 percent of the population. Since its inception, the primary care component of the mission has been under-prioritised relative to the hospital care component. This is clearly reflected in budgetary allocation trends which have favoured PMJAY over H\&W centres. Some have argued that H\&W centres are to mainly facilitate PMJAY and the business of empanelled private hospitals, while showing that the proposed target of 1.5 lakh H\&W centres by 2022 is unlikely to be fully accomplished (1). This under-emphasis on primary care under the dominant model of Indian UHC has been criticised across the board. Many have argued in favour of prioritising H\&W centres over PMJAY. Such arguments, however, have focused primarily on aspects related to health, economic, and efficiency gains - while the ethical aspect has been given little attention. This article discusses the ethical implications of the same. This becomes particularly crucial since there is currently an emerging consensus about expanding PMJAY to the remaining non-poor population, inspired in part by the Covid-19 pandemic (2).

\section{Priority setting and ethics}

UHC, which is conceptualised as access to quality health services for all without having to undergo financial hardship, is a highly ambitious goal. Despite moving forward with that ultimate goal in mind, LMICs cannot readily and reasonably provide coverage to everyone for all services owing to resource constraints. This makes it imperative to prioritise a set of "essential services" for coverage, which can then be expanded progressively as more resources become available. It becomes obvious that, since it involves crucial 
questions such as what and whom to prioritise, priority setting is inexorably connected with questions of fairness and ethics (3).

Daniels (4) identifies three essential components of priority setting while allocating scarce resources (4). First, resource allocation should be efficient, since health systems have a moral imperative to provide the maximum possible welfare with the available resources and thus, efficiency has not just economic but also ethical significance. Second, resource allocation must be fair and equitable, since health systems are not just concerned with maximising aggregate welfare but also about how such welfare is distributed. Third, since friction between these two concerns is ubiquitous, there should be a fair process of decision-making to resolve disputes. In line with the above, the World Health Organisation (WHO) Consultative Group on Equity and UHC lays down the criteria for determining priorities along all the three dimensions of UHC, namely population coverage, number of services covered, and financial risk protection (5). For service coverage, it suggests services to be classified into high, medium, and low-priority categories, with the highpriority services covered first. Three criteria are suggested for such categorisation, namely cost-effectiveness, priority to the worse off, and ability to provide financial risk protection.

George (6) has discussed how social determinants of health and public health services, which would qualify as highpriority in India, tend to be overridden by inpatient curative care due to an over-emphasis on financial risk protection (6). Here, I focus on the two components that currently dominate the Indian UHC discourse, namely primary care provision (H\&W centres) and inpatient care insurance (PMJAY).

\section{Skewed priorities}

Mukherjee (7) argues that more important than the amount of resources is the efficiency with which resources are allocated - and identifies seven cost effective areas for prioritisation under UHC in India (7), which fall largely under the ambit of primary care. Comprehensive primary care services indisputably fulfil the cost-effectiveness criterion of priority setting over inpatient care. They also fulfil the second criterion of "priority to the worse off", since the disadvantaged population is likely to be most benefited by an expansion of primary care services. The moot point then becomes financial risk protection - the third criterion that may be advanced as the prime justification for hospital insurance schemes like PMJAY. Here, it is important to note the widely known fact that outpatient consultation, diagnostics, and medicines, which are supposed to be offered free by $\mathrm{H} \& \mathrm{~W}$ centres, account for the majority share of out-of-pocket (OOP) spending on health. A purely quantitative comparison between OOP spending on inpatient and outpatient care may be inaccurate since they are often of different natures - outpatient care is financed mainly from current incomes while inpatient care comprises low frequency, high-impact episodes and often results in distress financing ie by borrowing money or selling assets. Nonetheless, in view of the substantial total share of outpatient care in OOP spending, its often pre-emptive effect on demand for inpatient care, and the additional costs imposed by hospitalisation (discussed later), primary care services score high in terms of financial risk protection. This makes them fulfil all the criteria necessary to be classified as "high-priority" services.

It is here that the prescriptions of the WHO Consultative Group (5) become important. It prescribes that "highpriority" services be expanded for everyone first, which in the Indian context would involve expansion of primary care services under the $\mathrm{H} \& \mathrm{~W}$ centre model. It classifies expansion of low- and medium-priority services before near-universal expansion of high-priority services as an "unacceptable trade-off 1", which is exactly what universal PMJAY expansion being currently considered would mean. The latter also fits into "unacceptable trade-off 2", which involves prioritising costly services, which offer fewer health benefits but substantial financial protection, over less costly alternatives that provide greater health benefits. Prioritising PMJAY over $\mathrm{H} \& \mathrm{~W}$ centres thus appears to be infringing the principles of fair UHC.

The WHO prescription of universal coverage of high-priority services first is in line with The Lancet Commission's Global Health 2035 (8) recommendation of progressive universalism. The commission's report posits universal coverage with certain essential services (even if the number of services is initially small) as being more pro-poor, equitable, and cost-effective than covering a large number of services for a smaller population or with substantial patient cost-sharing $(8,9)$. Vega and Frenz (10) consider the patterns of inequality in health service coverage by wealth quintiles across country contexts, and argue that for countries with significantly low coverage for all but the highest wealth quintile, a universal approach rather than a targeted approach is most cost-effective (10). Providing universal primary care through $\mathrm{H} \& \mathrm{~W}$ centres is thus the fair and ethical choice in the Indian context. On the contrary, prioritising PMJAY over H\&W centres artificially segregates the bottom two quintiles for coverage with services of lesser priority.

Ethical policy-making dictates that health systems cease to be mere health maximisers and make some provision for those in worse health and at risk of impoverishment due to the same. At any point of time, UHC should also include some arrangements for essential inpatient care needs and protection against large hospital bills. However, it is counterintuitive and unethical, particularly for low coverage health systems, to relegate to a secondary position primary care services, which offer large health gains equitably for a large population and also afford substantial financial protection by devoting a near exclusive focus on inpatient care services which are of last resort for individuals and households. Just 
as addressing social health determinants does more in the way of reducing avoidable mortality than medical determinants, primary care promises disproportionately greater health gains than last resort inpatient care. It also promises significant health and productivity gains across the life course $(9,11)$, which is particularly significant in the Indian context given its young population. The Lancet Commission resonates with this, as it proposes achievement of a "grand convergence" in health between poor and wealthy countries by investing in universal reduction of infectious, child, and maternal mortality rates to low levels (8). This has been identified as having a high return on investment.

It is thus clear that financial risk protection against sudden and large hospital expenses has been over-emphasised in the Indian case, to the extent that it has now become the main driver of the UHC agenda. This can be easily explained when we peruse the origins of PFHI in India on which our current vision of UHC is founded. PFHI received impetus with the launch of the Rajiv Arogyasri Scheme in united Andhra Pradesh in 2007, in response to petitions for protection against hospital expenses, which turned out to be a political success (12). Populist reduction of large treatment costs therefore inspires the current Indian vision of UHC. Apart from the populist rationale, there appears to be an added economic rationale in that the demand for hospital services is relatively inelastic and less prone to moral hazard. Notwithstanding this, an ethical rationale remains conspicuously missing.

\section{Other ethical dimensions}

There are a number of other ethical dimensions to the implementation and expansion of PMJAY in the Indian context. First, to be ethical, any programme needs to be based on sound evidence. The greater the costs and the limitations it imposes, the stronger the need for robust evidence (13). There is little evidence to support that PFHI provides significant financial risk protection. Evaluations of Rashtriya Swasthya Bima Yojana (RSBY) have shown that the scheme hardly had an impact on OOP spending and financial protection (14).

Second, there are glaring inequalities across the country in terms of healthcare institutions, infrastructure, and manpower - including the capacity to administer PMJAY itself. Such inequalities translate into inequities in access under insurance. What is even worse is that capacities are often the weakest in backward geographies where the need is most (15).

Third, PMJAY can significantly displace resources away from primary care oriented schemes like the National Health Mission. This is particularly concerning since overall increases in the health budget have been meagre. Further, PMJAY entails significant administrative costs, which do not primarily contribute towards improving health. Such costs are likely to increase manifold due to the contribution-based expansion of PMJAY to the "missing middle" population which the government is currently envisaging (2). In a setting of widespread unfulfilled basic health needs and scarce resources, every unnecessary rupee so incurred posits ethical questions (16).

Fourth, a hospitalisation episode can expose additional healthcare needs, which can increase the requirement for outpatient and support services (17). Further, hospitalisation entails significant indirect costs, such as lost wages and transportation expenses beyond the reimbursement limit, which are not covered under PFHI.

\section{The way forward}

There is need of systematic deliberation on the ethical dimensions of UHC in India, particularly with respect to the dominant model viz Ayushman Bharat. The fact that considerations of efficiency and effectiveness are integral to these ethical dimensions should provide greater momentum to such deliberation. This is particularly crucial in the current scenario where universal expansion of PMJAY is being envisaged, and ignoring considerations of ethics and fairness may "lock-in" certain undesirable traits which may be difficult to alter later on. There is need of a strong priority setting process with robust stakeholder participation to guide the rolling out of UHC in the country.

Rolling back an existing large-scale scheme like PMJAY could be politically and ethically contentious. Neither is the same warranted, since the scheme has certain desirable features that could help achieve UHC in India. However, any further expansion of the scheme to the non-poor population should be considered only after having adequately delivered on the primary care and population health agenda through the $\mathrm{H} \& \mathrm{~W}$ centres component. Even then, insurance expansion must be cautious and backed with sufficient evidence, especially considering the additional challenges involved in expanding contributory insurance to the non-poor informal sector. The public healthcare system should be bolstered to cater for the inpatient care needs of the non-poor population, through both traditional supply-side financing and additional PFHI revenues raised by public hospitals. With respect to the latter, addressing issues such as poor utilisation of insurance funds and high claims rejection rates in public hospitals would be crucial. The emerging evidence in this regard appears promising. Muraleedharan et al (18), in their analysis of the 71st and 75th NSSO data, demonstrate that utilisation of public sector services has increased, particularly among the non-poor, and OOP and catastrophic health expenditure in public facilities has declined (18). This has happened despite hardly any increase in per capita government expenditure, which is encouraging.

In 2019, the NITI Aayog released a roadmap entitled "Health System for a New India: Building Blocks" which emphasised reducing the fragmentation in the health financing, 
healthcare provider, and health information landscape. Today, about a year later, one can see initiatives like the National Digital Health Mission, and plans to integrate risk pools through PMJAY expansion, being promulgated. However, the bigger challenges of consolidating and streamlining outpatient care services and delivering on the unfinished public health agenda remain to be addressed. PMJAY and its expansion has huge cost implications in the face of sluggish health budgets. Under such circumstances, not prioritising comprehensive primary care would be a recipe of ensuring that the unfinished agenda remains unfinished indefinitely.

\section{Conflicts of interest and funding support: None declared.}

\section{References}

1. Hooda S. Decoding Ayushman Bharat. Econ Pol Wkly. 2020 Jun; 55(25):107-15.

2. Dey S. Govt to expand Ayushman Bharat to cover 'missing middle'. Times of India. 2020 Aug 15 [cited 2020 Sep 28]. Available from: https://timesofindia.indiatimes.com/india/govt-to-expandayushman-bharat-to-cover-missing-middle/articleshow/ 77555939.cms

3. Norheim OF. Ethical priority setting for universal health coverage: challenges in deciding upon fair distribution of health services. BMC Med. 2016; 14(75). https://doi.org/10.1186/s12916-016-0624-4

4. Daniels N. Resource allocation and priority setting. In: Barrett DH, Ortmann LH, Dawson A, Saenz C, Reis A, Bolan G, editors. Public Health Ethics: Cases Spanning the Globe. Springer Nature; 2016. pp. 61-94.

5. World Health Organization. Making fair choices on the path to universal health coverage: Final report of the WHO consultative group on equity and universal health coverage. Geneva: World Health Organization. 2014 [cited 2020 Sep 28]. Available from: https://apps.who.int/iris/handle/10665/112671
6. George M. Being fair in universal health coverage: prioritize public health services for low- and middle-income countries. Am J Public Health. 2016 May; 106(5):830-1.

7. Mukherjee K. Selective universalism: the paradoxical strategy to achieve universal health coverage in India. J Health Manag. 2019:21(1):154-9. Doi:10.1177/0972063418821826.

8. Jamison DT, Summers LH, Alleyne G, Arrow KJ, Berkley S, Binagwaho A, et al. Global health 2035: a world converging within a generation. Lancet. 2013 Dec 7;382(9908):1898-955. Doi: 10.1016/S01406736(13)62105-4.

9. Bloom DE, Khoury A, Subbaraman R. The promise and peril of universal health care. Science. 2018 Aug 24;361(6404):eaat9644. Doi: $10.1126 /$ science.aat9644.

10. Vega J, Frenz P. Integrating social determinants of health in the universal health coverage monitoring framework. Rev Panam Salud Publica. 2013 Dec;34(6):468-72.

11. Bleakley H. Malaria Eradication in the Americas: A Retrospective Analysis of Childhood Exposure. Am Econ J Appl Econ. 2010 Apr;2(2): 10.

12. Rao KS. Deconstructing Ayushman Bharat and Infusing Institutional Reform The Hindu Centre. 2018 Nov 20 [cited 2020 Sep 28]. Available from: https://www.thehinducentre.com/the-arena/current-issues/ article25545260.ece

13. Kass NE. An ethics framework for public health. Am J Public Health. 2001 Nov;91(11):1776-82.Doi: 10.2105/ajph.91.11.1776.

14. Ghosh S, Datta Gupta N. Targeting and effects of Rashtriya Swasthya Bima Yojana on access to care and financial protection. Econ Pol Wkly. 2017 Jan 28;52(4):61-70.

15. Lahariya C. 'Ayushman Bharat' program and universal health coverage in India. Indian Pediatr. 2018 Jun 15;55(6):495-506.

16. Bhaduri SD. A mix Indian health care can do without. Hindu. 2020 Feb 11 [cited 2020 Sep 28]. Available from: https:// www.thehindu.com/opinion/op-ed/a-mix-indian-health-care-cando-without/article30785823.ece

17. Zodpey S, Farooqui HH. Universal health coverage in India: progress achieved \& the way forward. Indian J Med Res. 2018 Apr;147(4):327329. Doi: 10.4103/ijmr.IJMR 616 18.

18. Muraleedharan VR, Vaidyanathan G, Sundararaman T, Dash U, Ranjan A, Rajesh M. Invest more in public healthcare facilities what do NSSO 71st and 75th rounds say? Econ Pol Wkly. 2020 Sep 12;55(37):53-60. 